\title{
A hybrid grey cuckoo search algorithm for job-shop scheduling problems under fuzzy conditions
}

\author{
Yang, F. ${ }^{a, b,{ }^{*}}$, Ye, C.M. ${ }^{a}$, Shi, M.H. ${ }^{a}$ \\ ${ }^{a}$ Business School, University of Shanghai for Science and Technology, Shanghai, P.R. China \\ ${ }^{b}$ College of Management, Henan University of Traditional Chinese Medicine, Zhengzhou, Henan, P.R. China
}

\begin{abstract}
A B S T R ACT
This paper aims to acquire the precise makespan or delivery period in jobshop scheduling (JSP) under fuzzy conditions. To this end, the author designed a grey scheduling model and a hybrid grey cuckoo search (HGCS) algorithm in the following steps. Firstly, three- and four-parameter interval grey numbers were introduced to depict the fuzzy makespan and delivery period, respectively; then, the possibility measure and necessity measure were defined, and the tardiness credibility index was proposed to estimate the probability of job tardiness. After that, a grey mixed integer programming model was developed to minimize the mean tardiness credibility, and the HGCS was proposed to solve the model. Finally, simulations were conducted on the classical example of $6(3) \times 6$. The results show that the proposed algorithm outperformed the basic cuckoo search. The research findings shed new light on the JSP under fuzzy conditions.
\end{abstract}

(c) 2018 CPE, University of Maribor. All rights reserved.

\author{
ARTICLE INFO \\ Keywords: \\ Job-shop scheduling problem \\ (JSP); \\ Grey scheduling; \\ Fuzzy condition; \\ Cuckoo search (CS); \\ Credibility; \\ Possibility measure; \\ Necessity measure \\ *Corresponding author: \\ yangfeng1126@126.com \\ (Yang, F.) \\ Article history: \\ Received 22 May 2018 \\ Revised 29 July 2018 \\ Accepted 22 August 2018
}

\section{References}

[1] Zhang, H., Liu, S., Moraca, S., Ojstersek, R. (2017). An effective use of hybrid metaheuristics algorithm for job shop scheduling problem, International Journal of Simulation Modelling, Vol. 16, No. 4, 644-657, doi: 10.2507/ IJSIMM16(4)7.400.

[2] Modrák, V., Pandian, R.S. (2010). Flow shop scheduling algorithm to minimize completion time for $n$-jobs $m$ machines problem, Tehnički vjesnik - Technical Gazette, Vol. 17, No. 3, 273-278.

[3] Chaudhry, I.A., Usman, M. (2017). Integrated process planning and scheduling using genetic algorithms, Tehnički vjesnik - Technical Gazette, Vol. 24, No. 5, 1401-1409, doi: 10.17559/TV-20151121212910.

[4] Huang, X.W., Zhao, X.Y., Ma, X.L. (2014). An improved genetic algorithm for job-shop scheduling problem with process sequence flexibility, International Journal of Simulation Modelling, Vol. 13, No. 4, 510-522, doi: 10.2507/ IJSIMM13(4)CO20.

[5] Ishii, H., Tada, M., Masuda, T. (1992). Two scheduling problems with fuzzy due-dates, Fuzzy Sets and Systems, Vol. 46, No. 3, 339-347, doi: 10.1016/0165-0114(92)90372-B.

[6] Noori-Darvish, S., Mahdavi, I., Mahdavi-Amiri, N. (2012). A bi-objective possibilistic programming model for open shop scheduling problems with sequence-dependent setup times, fuzzy processing times, and fuzzy due dates, Applied Soft Computing, Vol. 12, No. 4, 1399-1416, doi: 10.1016/i.asoc.2011.11.019.

[7] Gharehgozli, A.H., Tavakkoli-Moghaddam, R., Zaerpour, N. (2009). A fuzzy-mixed-integer goal programming model for a parallel-machine scheduling problem with sequence-dependent setup times and release dates, Robotics and Computer-Integrated Manufacturing, Vol. 25, No. 4-5, 853-859, doi: 10.1016/j.rcim.2008.12.005.

[8] Simeunović, N., Kamenko, I., Bugarski, V., Jovanović, M., Lalić, B. (2017). Improving workforce scheduling using artificial neural networks model, Advances in Production Engineering \& Management, Vol. 12, No. 4, 337-352, doi: $\underline{10.14743 / \text { apem2017.4.262. }}$. 
[9] Stanujkic, D., Magdalinovic, N., Jovanovic, R., Stojanovic, S. (2012). An objective multi-criteria approach to optimization using MOORA method and interval grey numbers, Technological and Economic Development of Economy, Vol. 18, No. 2, 331-363, doi: 10.3846/20294913.2012.676996.

[10] Zeng, B., Liu, S.-F., Xie, N.-M., Cui, J. (2010). Prediction model for interval grey number based on grey band and grey layer, Control and Decision, Vol. 25, No. 10, 1585-1588, doi: 10.13195/i.cd.2010.10.148.zengb.002.

[11] Luo, D., Wang, X. (2012). The multi-attribute grey target decision method for attribute value within threeparameter interval grey number, Applied Mathematical Modelling, Vol. 36, No. 5, 1957-1963, doi: 10.1016/ j.apm.2011.07.074.

[12] Luo, D. (2009). Decision-making methods with three-parameter interval grey number, Systems Engineering Theory \& Practice, Vol. 29, No. 1, 124-130, doi: 10.1016/s1874-8651(10)60033-6.

[13] Jin, F., Liu, P., Zhang, X. (2013). The multi-attribute group decision making method based on the interval grey linguistic variables weighted harmonic aggregation operators, Technological and Economic Development of Economy, Vol. 19, No. 3, 409-430, doi: 10.3846/20294913.2013.821685.

[14] Kamfiroozi, M.H., Aliahmadi, A., Jafari-Eskandari, M. (2012). Application of three parameter interval grey numbers in enterprise resource planning selection, International Journal of Information, Security and Systems Management, Vol. 1, No. 2, 72-77.

[15] Hu, Q., Zhang, W., Yu, L. (2007).The research and application of interval numbers of three parameters, Engineering Science, Vol. 9, No. 3, 47-51, doi: 10.3969/j.issn.1009-1742.2007.03.008.

[16] Sahu, N.K., Datta, S., Mahapatra, S.S. (2013). Decision making for selecting 3PL service provider using three parameter interval grey numbers, International Journal of Logistics Systems and Management, Vol. 14, No. 3, 261297, doi: 10.1504/ijlsm.2013.052061.

[17] Yang, B., Zhao, J. (2013). Correlation coefficients of hesitant three-parameter interval grey number and their applications to clustering analysis, Journal of Grey System, Vol. 25, No. 2, 139-147.

[18] Song, S. (2018). Application of gray prediction and linear programming model in economic management, Mathematical Modelling of Engineering Problems, Vol. 5, No. 1, 46-50, doi: 10.18280/mmep.050107.

[19] Luo, D. (2009). Decision-making methods with three-parameter interval grey number, Systems Engineering Theory \& Practice, Vol. 29, No. 1, 124-130, doi: 10.1016/s1874-8651(10)60033-6.

[20] Hu, J., Lin, Z. (2013). Multi-criteria decision making method based on interval numbers of four parameters, Operations Research and Management Science, Vol. 6, 84-91, doi: 10.3969/j.issn.1007-3221.2013.06.014.

[21] Wang, N., Hu, L.-P., Li, B.-J. (2014). Study on the distance entropy model based on three-parameter interval grey numbers and its application, Journal of Henan Agricultural University, Vol. 48, No. 3, 386-390.

[22] Zaccone, R., Sacile, R., Fossa, M. (2017). Energy modelling and decision support algorithm for the exploitation of biomass resources in industrial districts, International Journal of Heat and Technology, Vol. 35, No. S1 (Special Issue), S322-S329, doi: 10.18280/ijht.35Sp0144.

[23] Dai, Y., Zhu, X., Zhou, H., Mao, Z., Wu, W. (2018) Trajectory tracking control for seafloor tracked vehicle by adaptive neural-fuzzy inference system algorithm, International Journal of Computers Communications \& Control, Vol. 13, No. 4, 465-476, doi: 10.15837/ijccc.2018.4.3267.

[24] Wang, T.C., Xie, Y.Z. (2016). BP-GA data fusion algorithm studies oriented to smart home, Mathematical Modelling of Engineering Problems, Vol. 3, No. 3, 135-140.

[25] Tian, Y., Chen, W., Li, L., Wang, X., Liu, Z. (2018). Gait recognition via coalitional game-based feature selection and extreme learning machine, NeuroQuantology, Vol. 16, No. 2, 32-39, doi: 10.14704/nq.2018.16.2.1173.

[26] Xu, H., Bao, Z.R., Zhang, T. (2017). Solving dual flexible job-shop scheduling problem using a Bat Algorithm, Advances in Production Engineering \& Management, Vol. 12, No. 1, 5-16, doi: 10.14743/apem2017.1.235.

[27] Yang, X.-S., Deb, S. (2010). Cuckoo search via Lévy flights, In: 2009 World Congress on Nature \& Biologically Inspired Computing (NaBIC), Coimbatore, India, 210-214, doi: 10.1109/NABIC.2009.5393690.

[28] Liang, C.H., Zeng, S., Li, Z.X., Yang, D.G., Sherif, S.A. (2016). Optimal design of plate-fin heat sink under natural convection using a particle swarm optimization algorithm, International Journal of Heat \& Technology, Vol. 34, No. 2, 275-280, doi: $10.18280 /$ ijht.340217.

[29] Sen, G.D., Sharma, J., Goyal, G.R., Singh, A.K. (2017). A multi-objective PSO (MOPSO) algorithm for optimal active power dispatch with pollution control, Mathematical Modelling of Engineering Problems, Vol. 4, No. 3, 113-119, doi: $10.18280 / \mathrm{mmep} .040301$.

[30] Yang, X.-S., Deb, S. (2013). Multiobjective cuckoo search for design optimization, Computers \& Operations Research, Vol. 40, No. 6, 1616-1624, doi: 10.1016/i.cor.2011.09.026.

[31] Valian, E., Mohanna, S., Tavakoli, S. (2011). Improved cuckoo search algorithm for feedforward neural network training, International Journal of Artificial Intelligence \& Applications, Vol. 2, No. 3, 36-43, doi: 10.5121/ijaia. 2011.2304.

[32] Walton, S., Hassan, O., Morgan, K., Brown, M.R. (2011). Modified cuckoo search: A new gradient free optimisation algorithm, Chaos, Solitons \& Fractals, Vol. 44, No. 9, 710-718, doi: 10.1016/j.chaos.2011.06.004. 
APEM
ISSN 1854-6250

Spletna stran: apem-journal.org Izvirni znanstveni članek

\section{Hibridni sivi algoritem kukavičjega iskanja za terminiranja proizvodnje po naročilu $v$ nejasnih pogojih}

Yang, F. ${ }^{a, b,}{ }^{,}$, Ye, C.M. ${ }^{a}$, Shi, M.H. ${ }^{a}$

${ }^{a}$ Business School, University of Shanghai for Science and Technology, Shanghai, P.R. China

${ }^{b}$ College of Management, Henan University of Traditional Chinese Medicine, Zhengzhou, Henan, P.R. China

\section{POVZETEK}

Namen prispevka je pridobiti natančen čas izdelave ali čas dobave izdelka za problem terminiranja proizvodnje po naročilu v nejasnih pogojih. Za ta namen smo zasnovali siv model načrtovanja in hibridni algoritem kukavičjega iskanja (HGCS) v naslednjih korakih. Najprej so bile vpeljane tri- in štiriparametrične sive intervalne številke, ki predstavljajo mehki čas izdelave in mehki čas dobave izdelka. Nato sta bila opredeljena možnost ukrepanja ter nujnost ukrepanja, predlagan pa je bil tudi indeks verodostojnosti za oceno verjetnosti izvedbe proizvodnega procesa. Nadalje je bil razvit siv mešani celoštevilski programski model. Za zmanjšanje povprečne verjetnosti neizvedbe proizvodnega procesa je bil uporabljen HGCS algoritem. Nato so bile izvedene simulacije na klasičnem $6(3) \times 6$ primeru. Rezultati kažejo, da je predlagani algoritem presegel sposobnosti osnovnega algoritma kukavičjega iskanja. Ugotovitve raziskave prikazujejo problem terminiranja proizvodnje po naročilu v nejasnih pogoji v novi luči.

(c) 2018 CPE, University of Maribor. All rights reserved.

\section{PODATKI O ČLANKU}

Ključne besede:

Problem terminiranja proizvodnje po naročilu (JSP);

Sivo terminiranje;

Nejasni pogoji;

Kukavičje iskanje (CS);

Verodostojnost;

Merilo možnosti;

Merilo nujnosti

*Kontaktna oseba:

yangfeng1126@126.com

(Yang, F.)

Zgodovina članka:

Prejet 22. maja 2018

Popravljen 29. julija 2018

Sprejet 22. avgusta 2018 